\title{
2-LOCAL DERIVATIONS ON WITT ALGEBRAS
}

\author{
YUEQIANG ZHAO, YANG CHEN, AND KAIMING ZHAO
}

\begin{abstract}
In this paper, we prove that every 2-local derivation on Witt algebras $W_{n}, W_{n}^{+}$or $W_{n}^{++}$is a derivation for all $n=1,2, \cdots, \infty$. As a consequence we obtain that every 2-local derivation on any centerless generalized Virasoro algebra of higher rank is a derivation.
\end{abstract}

Keywords: Lie algebra, Witt algebra, derivation, 2-local derivation. AMS Subject Classification: 17B05, 17B40, $17 \mathrm{~B} 66$.

\section{INTRODUCTION}

In 1997, Šemrl [11] introduced the notion of 2-local derivations on algebras. Namely, for an associative algebra $A$, a map $\Delta: A \rightarrow A$ (not necessarily linear) is called a 2-local derivation if for every $x, y \in A$, there exists a derivation $D_{x, y}: A \rightarrow A$ such that $\Delta(x)=D_{x, y}(x)$ and $\Delta(y)=D_{x, y}(y)$. The concept of 2-local derivation is actually an important and interesting property for an algebra. Recently, several papers have been devoted to similar notions and corresponding problems for Lie (super)algebras $L$. The main problem in this subject is to determine all 2-local derivations, and to see whether 2-local derivations automatically become a derivation of $L$. In [1] it is proved that every 2-local derivation on a finite-dimensional semi-simple Lie algebra $L$ over complex numbers is a derivation and that each finite-dimensional nilpotent Lie algebra with dimension larger than two admits a 2-local derivation which is not a derivation. In [12] and [2] Ayupov and Yusupov studied 2-local derivations on univariate Witt algebras. In the present paper we study 2-local derivations on multivariate Witt algebras.

The Witt algebra $W_{n}$ of vector fields on an $n$-dimensional torus is the derivation Lie algebra of the Laurent polynomial algebra $A_{n}=\mathbb{C}\left[t_{1}^{ \pm 1}, t_{2}^{ \pm 1}, \cdots, t_{n}^{ \pm 1}\right]$. Witt algebras were one of the four classes of Cartan type Lie algebras originally introduced in 1909 by Cartan [6] when he studied infinite dimensional simple Lie algebras. Over the last two decades, the representation theory of Witt algebras was extensively studied by many mathematicians and physicists; see for example [4,5, 9]. Very recently, Billig and Futorny [4] obtained the classification for all simple Harish-Chandra $W_{n}$-modules.

The paper is organized as follows. In Section 2 we recall some known results and establish some related properties concerning Witt algebras and prove an auxiliary result for later use. In Section 3 we prove that every 2-local derivation on Witt algebras $W_{n}$ is a derivation. As a consequence we obtain that every 2-local derivation on any centerless generalized Virasoro algebra of higher rank is a derivation. In Section 4 we obtain similar results for $W_{\infty}$. Finally, in Section 5 we show that the above methods and conclusions are applicable for Witt algebras $W_{n}^{+}, W_{n}^{++}, W_{\infty}^{+}$and $W_{\infty}^{++}$.

Throughout this paper, we denote by $\mathbb{Z}, \mathbb{N}, \mathbb{Z}_{+}$and $\mathbb{C}$ the sets of all integers, positive integers, non-negative integers and complex numbers respectively. All algebras are over $\mathbb{C}$. 


\section{The Witt Algebras}

In this section we recall definitions, symbols and establish some auxiliary results for later use in this paper.

A derivation on a Lie algebra $L$ is a linear map $D: L \rightarrow L$ which satisfies the Leibniz law

$$
D([x, y])=[D(x), y]+[x, D(y)], \forall x, y \in L .
$$

The set of all derivations of $L$ is a Lie algebra and is denoted by $\operatorname{Der}(L)$. For any $a \in L$, the map

$$
\operatorname{ad}(a): L \rightarrow L, \operatorname{ad}(a) x=[a, x], \forall x \in L
$$

is a derivation and derivations of this form are called inner derivations. The set of all inner derivations of $L$, denoted by $\operatorname{Inn}(L)$, is an ideal in $\operatorname{Der}(L)$. A derivation $D$ of $L$ is a locally inner derivation if for any finite subset $S$ of $L$ there exist a finite-dimensional subspace $V$ of $L$ containing $S$ and $a \in L$ such that $\left.D\right|_{V}=\left.\operatorname{ad}(a)\right|_{V}$.

For $n \in \mathbb{N}$, let $A_{n}=\mathbb{C}\left[t_{1}^{ \pm 1}, t_{2}^{ \pm 1}, \cdots, t_{n}^{ \pm 1}\right]$ be the Laurent polynomial algebra and $W_{n}=\operatorname{Der}\left(A_{n}\right)$ be the Witt algebra of vector fields on an $n$-dimensional torus. Thus $W_{n}$ has a natural structure of an $A_{n}$-module, which is free of rank $n$. We choose $d_{1}=t_{1} \frac{\partial}{\partial t_{1}}, \ldots, d_{n}=t_{n} \frac{\partial}{\partial t_{n}}$ as a basis of this $A_{n}$-module:

$$
W_{n}=\bigoplus_{i=1}^{n} A_{n} d_{i}
$$

Denote $t^{\alpha}=t_{1}^{\alpha_{1}} \cdots t_{n}^{\alpha_{n}}$ for $\alpha=\left(\alpha_{1}, \ldots, \alpha_{n}\right) \in \mathbb{Z}^{n}$ and let $\left\{\epsilon_{1}, \ldots, \epsilon_{n}\right\}$ be the standard basis of $\mathbb{Z}^{n}$. Then we can write the Lie bracket in $W_{n}$ as follows:

$$
\left[t^{\alpha} d_{i}, t^{\beta} d_{j}\right]=\beta_{i} t^{\alpha+\beta} d_{j}-\alpha_{j} t^{\alpha+\beta} d_{i}, i, j=1, \ldots, n ; \alpha, \beta \in \mathbb{Z}^{n} .
$$

The subspace $\mathfrak{h}$ spanned by $d_{1}, \ldots, d_{n}$ is the Cartan subalgebra in $W_{n}$. We may write any nonzero element in $W_{n}$ as $\sum_{\alpha \in S} t^{\alpha} d_{\alpha}$, where $S$ is the finite subset consisting of all $\alpha \in \mathbb{Z}^{n}$ with $d_{\alpha} \in \mathfrak{h} \backslash\{0\}$. Suppose that $d_{\alpha}=c_{1} d_{1}+\cdots+c_{n} d_{n} \in \mathfrak{h}$ and $\beta \in \mathbb{Z}^{n}$, let

$$
\left(d_{\alpha}, \beta\right)=c_{1} \beta_{1}+\cdots+c_{n} \beta_{n} .
$$

Then we get the following formula

$$
\left[t^{\alpha} d_{\alpha}, t^{\beta} d_{\beta}\right]=t^{\alpha+\beta}\left(\left(d_{\alpha}, \beta\right) d_{\beta}-\left(d_{\beta}, \alpha\right) d_{\alpha}\right) .
$$

Denote by $\mathbb{Z}^{\infty}$ (resp. $\left.\mathbb{Z}_{+}^{\infty}, \mathbb{C}^{\infty}\right)$ the set of all infinite sequences $\alpha=\left(\alpha_{1}, \alpha_{2}, \alpha_{3}, \cdots\right)$ where $\alpha_{i} \in \mathbb{Z}$ (resp. $\mathbb{Z}_{+}, \mathbb{C}$ ) and only a finite number of $\alpha_{i}$ can be nonzero. We can obtain similar definition and formula for the Witt algebra $W_{\infty}=\operatorname{Der}\left(\mathbb{C}\left[t_{1}^{ \pm 1}, t_{2}^{ \pm 1}, \cdots\right]\right)$.

Definition 2.1. We call a vector $\mu=\left(\mu_{1}, \mu_{2}, \cdots, \mu_{n}\right) \in \mathbb{C}^{n}$ generic if

$$
\mu \cdot \alpha=\alpha_{1} \mu_{1}+\alpha_{2} \mu_{2}+\cdots+\alpha_{n} \mu_{n} \neq 0, \forall \alpha=\left(\alpha_{1}, \alpha_{2}, \cdots, \alpha_{n}\right) \in \mathbb{Z}^{n} \backslash\{0\} .
$$

Note that a vector $\mu=\left(\mu_{1}, \mu_{2}, \cdots, \mu_{n}\right) \in \mathbb{C}^{n}$ is generic if and only if $\mu_{1}, \mu_{2}, \cdots, \mu_{n}$ are linearly independent over the rational numbers $\mathbb{Q}$. There are always generic vectors. For example, $\mu=\left(\zeta, \zeta^{2}, \ldots, \zeta^{n}\right) \in \mathbb{C}^{n}$ is just a generic vector, where $\zeta$ is a algebraic number of degree more than $n$ or a transcendental number in $\mathbb{C}$. Let $\mu$ be a generic vector in $\mathbb{C}^{n}$ and $d_{\mu}=\mu_{1} d_{1}+\cdots+\mu_{n} d_{n}$. Then we have the Lie subalgebra of $W_{n}$ :

$$
W_{n}(\mu)=A_{n} d_{\mu}
$$

which is called (centerless) generalized Virasoro algebra of rank $n$, see [10].

We proof the following key lemma at first. 
Lemma 2.2. For any $k \in \mathbb{Z} \backslash\{0\}$, the centralizer of $\left(t_{1}^{k}+\cdots+t_{n}^{k}\right) d_{\mu}$ in $W_{n}$ is $\mathbb{C}\left(t_{1}^{k}+\cdots+t_{n}^{k}\right) d_{\mu}$.

Proof. We firstly deal with the case $k>0$. Let $x \in W_{n} \backslash\{0\}$ such that $\left[x,\left(t_{1}^{k}+\cdots+\right.\right.$ $\left.\left.t_{n}^{k}\right) d_{\mu}\right]=0$. We may write

$$
x=\sum_{\alpha \in S} t^{\alpha} d_{\alpha}
$$

where $S$ is the finite subset consisting of all $\alpha \in \mathbb{Z}^{n}$ with $d_{\alpha} \in \mathfrak{h} \backslash\{0\}$. Take a term $t^{\beta} d_{\beta}$ of maximal degree with respect to some $t_{i}$ in the right-hand-side of (2.1). Suppose $\beta \neq 0$ (if it exists).

Claim 1. We must have $t^{\beta} d_{\beta}=c_{i} t_{i}^{k} d_{\mu}$ for some $c_{i} \in \mathbb{C}$.

The term $\left[t^{\beta} d_{\beta}, t_{i}^{k} d_{\mu}\right]$ is of maximal degree with respect to $t_{i}$ in $\left[x,\left(t_{1}^{k}+\cdots+t_{n}^{k}\right) d_{\mu}\right]$. We must have

$$
\left[t^{\beta} d_{\beta}, t_{i}^{k} d_{\mu}\right]=t^{\beta+k \epsilon_{i}}\left(\left(d_{\beta}, k \epsilon_{i}\right) d_{\mu}-\left(d_{\mu}, \beta\right) d_{\beta}\right)=0 .
$$

Since $\left(d_{\mu}, \beta\right) \neq 0$, we have $d_{\beta}=c_{i} d_{\mu}$ for some $0 \neq c_{i} \in \mathbb{C}$ and furthermore

$$
\left(d_{\beta}, k \epsilon_{i}\right) d_{\mu}-\left(d_{\mu}, \beta\right) d_{\beta}=\left(c_{i} d_{\mu}, k \epsilon_{i}\right) d_{\mu}-\left(d_{\mu}, \beta\right) c_{i} d_{\mu}=c_{i}\left(d_{\mu}, k \epsilon_{i}-\beta\right) d_{\mu}=0 .
$$

So $\beta=k \epsilon_{i}$. Claim 1 follows.

Now $c_{i} t_{i}^{k} d_{\mu}$ is the only possible term of maximal degree with respect to $t_{i}$ in (2.1). If there is such a term, we consider $c_{1} t_{1}^{k} d_{\mu}$ without loss of generality. For $i \neq 1$, to delete the term $\left[c_{1} t_{1}^{k} d_{\mu}, t_{i}^{k} d_{\mu}\right]=c_{1} k\left(\mu_{i}-\mu_{1}\right) t_{1}^{k} t_{i}^{k} d_{\mu} \neq 0$ in $\left[x,\left(t_{1}^{k}+\cdots+t_{n}^{k}\right) d_{\mu}\right]$, there must be the term $c_{1} t_{i}^{k} d_{\mu}$ in (2.1). Thus $c_{i}=c_{1}$ for all $1 \leq i \leq n$. Let $x^{\prime}=x-c_{1}\left(t_{1}^{k}+\cdots+t_{n}^{k}\right) d_{\mu}$. Then $\left[x^{\prime},\left(t_{1}^{k}+\cdots+t_{n}^{k}\right) d_{\mu}\right]=0$. Applying Claim 1 to $x^{\prime}$, we see that the term $d$ of maximal degree with respect to any $t_{i}$ in $x^{\prime}$ is in the Cartan subalgebra $\mathfrak{h}$. Therefore

$$
\left[d, t_{i}^{k} d_{\mu}\right]=\left(d, k \epsilon_{i}\right) t_{i}^{k} d_{\mu}=0, \forall i=1, \ldots, n .
$$

We obtain that $d=0$ and must have $x^{\prime}=0$. For $k<0$, we may take terms of minimal degree instead. So for any $k \in \mathbb{Z} \backslash\{0\}$, the centralizer of $\left(t_{1}^{k}+\cdots+t_{n}^{k}\right) d_{\mu}$ is only $\mathbb{C}\left(t_{1}^{k}+\cdots+t_{n}^{k}\right) d_{\mu}$.

From Proposition 4.1 and Theorem 4.3 in [7] we know that any derivation on $W_{n}$ (resp. $W_{\infty}$ ) is inner (resp. locally inner). Then for the Witt algebra $W=W_{n}$ or $W_{\infty}$ the above definition of the 2-local derivation can be reformulated as follows. A map $\Delta$ on $W$ is a 2-local derivation on $W$ if for any two elements $x, y \in W$ there exists an element $a_{x, y} \in W$ such that

$$
\Delta(x)=\left[a_{x, y}, x\right], \quad \Delta(y)=\left[a_{x, y}, y\right] .
$$

In general, a 2-local derivation $\Delta$ on a Lie algebra $L$ is a derivation if for any two elements $x, y \in L$ and $c \in \mathbb{C}$ we have

$$
\Delta(x+c y)=\Delta(x)+c \Delta(y), \Delta([x, y])=[\Delta(x), y]+[x, \Delta(y)] .
$$

\section{2-LOCAL DERIVATIONS ON $W_{n}$}

Now we shall give the main result concerning 2-local derivations on $W_{n}$.

Theorem 3.1. Every 2-local derivation on the Witt algebra $W_{n}$ is a derivation.

For the proof of this Theorem we need to set up several Lemmas as preparations. Let $\mu$ be a generic vector in $\mathbb{C}^{n}$ and $d_{\mu}=\mu_{1} d_{1}+\cdots+\mu_{n} d_{n}$. 
Lemma 3.2. Let $\Delta$ be a 2-local derivation on $W_{n}$ such that $\Delta\left(d_{\mu}\right)=0$. Then for any nonzero element $x=\sum_{\alpha \in S} t^{\alpha} d_{\alpha} \in W_{n}$, where $S$ is the finite subset consisting of all $\alpha \in \mathbb{Z}^{n}$ with $d_{\alpha} \in \mathfrak{h} \backslash\{0\}$, we have $\Delta(x)=\Delta\left(\sum_{\alpha \in S} t^{\alpha} d_{\alpha}\right) \in \sum_{\alpha \in S} \mathbb{C} t^{\alpha} d_{\alpha}$.

Proof. For $x$ and $d_{\mu}$, there exists an element $a=\sum_{\beta \in \mathbb{Z}^{n}} t^{\beta} d_{\beta} \in W_{n}$, such that

$$
\Delta\left(d_{\mu}\right)=\left[a, d_{\mu}\right], \Delta(x)=\Delta\left(\sum_{\alpha \in S} t^{\alpha} d_{\alpha}\right)=\left[a, \sum_{\alpha \in S} t^{\alpha} d_{\alpha}\right] .
$$

Then

$$
0=\Delta\left(d_{\mu}\right)=\left[a, d_{\mu}\right]=\left[\sum_{\beta \in \mathbb{Z}^{n}} t^{\beta} d_{\beta}, d_{\mu}\right]=-\sum_{\beta \in \mathbb{Z}^{n}} t^{\beta}\left(d_{\mu}, \beta\right) d_{\beta} .
$$

This means that $a=d_{0} \in \mathfrak{h}$. Thus

$$
\Delta(x)=\Delta\left(\sum_{\alpha \in S} t^{\alpha} d_{\alpha}\right)=\left[d_{0}, \sum_{\alpha \in S} t^{\alpha} d_{\alpha}\right] \in \sum_{\alpha \in S} \mathbb{C} t^{\alpha} d_{\alpha} .
$$

Lemma 3.3. Let $\Delta$ be a 2-local derivation on $W_{n}$ such that

$$
\Delta\left(d_{\mu}\right)=\Delta\left(\left(t_{1}+\cdots+t_{n}\right) d_{\mu}\right)=0 .
$$

Then $\Delta\left(\left(t_{1}^{k}+\cdots+t_{n}^{k}\right) d_{\mu}\right)=0$ for all $k \in \mathbb{Z}$.

Proof. Suppose $k \neq 0,1$. For $\left(t_{1}+\cdots+t_{n}\right) d_{\mu}$ and $\left(t_{1}^{k}+\cdots+t_{n}^{k}\right) d_{\mu}$, there exists an element $a \in W_{n}$ such that

$$
\begin{gathered}
0=\Delta\left(\left(t_{1}+\cdots+t_{n}\right) d_{\mu}\right)=\left[a,\left(t_{1}+\cdots+t_{n}\right) d_{\mu}\right], \\
\Delta\left(\left(t_{1}^{k}+\cdots+t_{n}^{k}\right) d_{\mu}\right)=\left[a,\left(t_{1}^{k}+\cdots+t_{n}^{k}\right) d_{\mu}\right] .
\end{gathered}
$$

Then $a=c\left(t_{1}+\cdots+t_{n}\right) d_{\mu}$ for some $c \in \mathbb{C}$ by Lemma 2.2. Using Lemma 3.2 we have

$$
\Delta\left(\left(t_{1}^{k}+\cdots+t_{n}^{k}\right) d_{\mu}\right)=\left[c\left(t_{1}+\cdots+t_{n}\right) d_{\mu},\left(t_{1}^{k}+\cdots+t_{n}^{k}\right) d_{\mu}\right] \in \sum_{i=1}^{n} \mathbb{C} t_{i}^{k} d_{\mu} .
$$

It implies that $c=0$, otherwise there exists a nonzero term $c(k-1) \mu_{1} t_{1}^{k+1} d_{\mu}$ in $\Delta\left(\left(t_{1}^{k}+\right.\right.$ $\left.\left.\cdots+t_{n}^{k}\right) d_{\mu}\right)$ but not in $\sum_{i=1}^{n} \mathbb{C} t_{i}^{k} d_{\mu}$. Hence $\Delta\left(\left(t_{1}^{k}+\cdots+t_{n}^{k}\right) d_{\mu}\right)=0$ for all $k \in \mathbb{Z}$.

Lemma 3.4. Let $\Delta$ be a 2-local derivation on $W_{n}$ such that $\Delta\left(\left(t_{1}^{k}+\cdots+t_{n}^{k}\right) d_{\mu}\right)=0$ for all $k \in \mathbb{Z}$. Then $\Delta=0$.

Proof. Take an arbitrary nonzero element $x=\sum_{\alpha \in S} t^{\alpha} d_{\alpha} \in W_{n}$, where $S$ is the finite subset consisting of all $\alpha \in \mathbb{Z}^{n}$ with $d_{\alpha} \in \mathfrak{h} \backslash\{0\}$. Let $n_{x}$ be an index such that $\left|\alpha_{i}\right|<n_{x}$ for any $\alpha \in S$. For the fixed $k>2 n_{x}$, there is an element $a \in W_{n}$ such that

$$
\begin{gathered}
0=\Delta\left(\left(t_{1}^{k}+\cdots+t_{n}^{k}\right) d_{\mu}\right)=\left[a,\left(t_{1}^{k}+\cdots+t_{n}^{k}\right) d_{\mu}\right], \\
\Delta\left(\sum_{\alpha \in S} t^{\alpha} d_{\alpha}\right)=\left[a, \sum_{\alpha \in S} t^{\alpha} d_{\alpha}\right] .
\end{gathered}
$$

Then $a=c\left(t_{1}^{k}+\cdots+t_{n}^{k}\right) d_{\mu}$ for some $c \in \mathbb{C}$ by Lemma 2.2. Using Lemma 3.2, we have

$$
\Delta\left(\sum_{\alpha \in S} t^{\alpha} d_{\alpha}\right)=\left[c\left(t_{1}^{k}+\cdots+t_{n}^{k}\right) d_{\mu}, \sum_{\alpha \in S} t^{\alpha} d_{\alpha}\right] \in \sum_{\alpha \in S} \mathbb{C} t^{\alpha} d_{\alpha} .
$$

It implies that $c=0$, otherwise there exists some $t_{i}$ whose degree is more than $n_{x}$ for any term in $\Delta\left(\sum_{\alpha \in S} t^{\alpha} d_{\alpha}\right)$. So $\Delta=0$. 
Now we are in position to prove Theorem 3.1

Proof of Theorem 3.1 Let $\Delta$ be a 2-local derivation on $W_{n}$. Take an element $a \in W_{n}$ such that

$$
\Delta\left(d_{\mu}\right)=\left[a, d_{\mu}\right], \Delta\left(\left(t_{1}+\cdots+t_{n}\right) d_{\mu}\right)=\left[a,\left(t_{1}+\cdots+t_{n}\right) d_{\mu}\right] .
$$

Set $\Delta_{1}=\Delta-\operatorname{ad}(a)$. Then $\Delta_{1}$ is a 2-local derivation such that

$$
\Delta_{1}\left(d_{\mu}\right)=\Delta_{1}\left(\left(t_{1}+\cdots+t_{n}\right) d_{\mu}\right)=0 .
$$

By Lemma 3.3 and Lemma 3.4, it follows that $\Delta_{1}=0$. Thus $\Delta=\operatorname{ad}(a)$ is a derivation. The proof is complete.

By Theorem 3.4 in [8] any derivation on the generalized Virasoro algebra $W_{n}(\mu)$ can be seen as the restriction of a inner derivation on $W_{n}$. All the proofs in this section with minor modifications are valid for the generalized Virasoro algebra $W_{n}(\mu)$. Therefore we obtain the following consequence.

Corollary 3.5. Let $n \in \mathbb{N}$, and let $\mu \in \mathbb{C}^{n}$ be generic. Then any 2-local derivation on the generalized Virasoro algebra $W_{n}(\mu)$ is a derivation.

\section{2-LOCAL DERIVATIONS ON $W_{\infty}$}

In this section we shall determine all 2-local derivations on $W_{\infty}$. For a given $n \in \mathbb{N}$, we know that $W_{n}=\operatorname{Der}\left(\mathbb{C}\left[t_{1}^{ \pm 1}, t_{2}^{ \pm 1}, \cdots, t_{n}^{ \pm 1}\right]\right)$ is a subalgebra of $W_{\infty}=\operatorname{Der}\left(\mathbb{C}\left[t_{1}^{ \pm 1}, t_{2}^{ \pm 1}, \cdots\right]\right)$. We still suppose that $\mu$ is a generic vector in $\mathbb{C}^{n}$ and $d_{\mu}=\mu_{1} d_{1}+\cdots+\mu_{n} d_{n}$. The Cartan subalgebra of $W_{\infty}\left(\right.$ resp. $\left.W_{n}\right)$ is denoted by $\mathfrak{h}_{\infty}\left(\right.$ resp. $\left.\mathfrak{h}_{n}\right)$. For convenience we define

$$
\begin{aligned}
& K_{n}=\left\{\alpha \in \mathbb{Z}^{\infty}: \alpha_{1}=\alpha_{2}=\cdots=\alpha_{n}=0\right\}, \\
& \mathfrak{h}_{n}^{\prime}=\left\{h \in \mathfrak{h}_{\infty}:\left(h, \epsilon_{i}\right)=0 \forall i=1,2, \cdots, n\right\} .
\end{aligned}
$$

Lemma 4.1. For a given $n \in \mathbb{N}$ and any $k \in \mathbb{Z} \backslash\{0\}$, the centralizer of $\left(t_{1}^{k}+\cdots+t_{n}^{k}\right) d_{\mu}$ in $W_{\infty}$ is

$$
\sum_{\beta \in K_{n}} \mathbb{C} t^{\beta}\left(t_{1}^{k}+\cdots+t_{n}^{k}\right) d_{\mu}+\sum_{\beta \in K_{n}} t^{\beta} \mathfrak{h}_{n}^{\prime}
$$

Proof. We firstly deal with the case $k>0$. Let $x \in W_{\infty} \backslash\{0\}$ such that $\left[x,\left(t_{1}^{k}+\cdots+\right.\right.$ $\left.\left.t_{n}^{k}\right) d_{\mu}\right]=0$. We may write

$$
x=\sum_{\alpha \in S} t^{\alpha} d_{\alpha}
$$

where $S$ is the finite subset consisting of all $\alpha \in \mathbb{Z}^{\infty}$ with $d_{\alpha} \in \mathfrak{h}_{\infty} \backslash\{0\}$. Take a term $t^{\gamma} d_{\gamma}$ of maximal degree with respect to some $t_{i}(1 \leq i \leq n)$ in the right-hand-side of (4.1). Suppose that $\gamma \notin K_{n}$ (if it exists).

Claim 1. We must have $t^{\gamma} d_{\gamma}=c_{\beta}^{(i)} t^{\beta+k \epsilon_{i}} d_{\mu}$ for some $\beta \in K_{n}$ and $c_{\beta}^{(i)} \in \mathbb{C}$. So

The term $\left[t^{\gamma} d_{\gamma}, t_{i}^{k} d_{\mu}\right]$ is of maximal degree with respect to $t_{i}$ in $\left[x,\left(t_{1}^{k}+\cdots+t_{n}^{k}\right) d_{\mu}\right]$.

$$
\left[t^{\gamma} d_{\gamma}, t_{i}^{k} d_{\mu}\right]=t^{\gamma+k \epsilon_{i}}\left(\left(d_{\gamma}, k \epsilon_{i}\right) d_{\mu}-\left(d_{\mu}, \gamma\right) d_{\gamma}\right)=0
$$

Since $\left(d_{\mu}, \gamma\right) \neq 0$, we deduce that $d_{\gamma}=c_{\beta}^{(i)} d_{\mu}, 0 \neq c_{\beta}^{(i)} \in \mathbb{C}$, yielding that

$$
\left(d_{\gamma}, k \epsilon_{i}\right) d_{\mu}-\left(d_{\mu}, \gamma\right) d_{\gamma}=\left(c_{\beta}^{(i)} d_{\mu}, k \epsilon_{i}\right) d_{\mu}-\left(d_{\mu}, \gamma\right) c_{\beta}^{(i)} d_{\mu}=c_{\beta}^{(i)}\left(d_{\mu}, k \epsilon_{i}-\gamma\right) d_{\mu}=0 .
$$

We see that $\gamma=\beta+k \epsilon_{i}$ for some $\beta \in K_{n}$. Claim 1 follows. 
Now a finite sum $\sum_{\beta \in K_{n}} c_{\beta}^{(i)} t^{\beta} t_{i}^{k} d_{\mu}$, where $c_{\beta}^{(i)} \in \mathbb{C}$, involves the only possible terms of maximal degree with respect to $t_{i}$ in (4.1). If there is such a finite sum, we consider $\sum_{\beta \in K_{n}} c_{\beta}^{(1)} t^{\beta} t_{1}^{k} d_{\mu}$ without loss of generality. Hence, to delete the terms

$$
\left[\sum_{\beta \in K_{n}} c_{\beta}^{(1)} t^{\beta} t_{1}^{k} d_{\mu}, t_{i}^{k} d_{\mu}\right]=\sum_{\beta \in K_{n}} c_{\beta}^{(1)} k\left(\mu_{i}-\mu_{1}\right) t^{\beta} t_{1}^{k} t_{i}^{k} d_{\mu} \neq 0, i \neq 1,
$$

there must be the terms $\sum_{\beta \in K_{n}} c_{\beta}^{(1)} t^{\beta} t_{i}^{k} d_{\mu}$ in (4.1). Thus $c_{\beta}^{(i)}=c_{\beta}^{(1)}$ for all $1 \leq i \leq n$. Let

$$
x^{\prime}=x-\sum_{\beta \in K_{n}} c_{\beta}^{(1)} t^{\beta}\left(t_{1}^{k}+\cdots+t_{n}^{k}\right) d_{\mu} .
$$

Then $\left[x^{\prime},\left(t_{1}^{k}+\cdots+t_{n}^{k}\right) d_{\mu}\right]=0$. Applying Claim 1 to $x^{\prime}$, we see that the terms of maximal degree with respect to any $t_{i}(1 \leq i \leq n)$ in $x^{\prime}$ are $\sum_{\beta \in K_{n}} t^{\beta} d_{\beta}, d_{\beta} \in \mathfrak{h}_{\infty}$. Therefore

$$
\left[\sum_{\beta \in K_{n}} t^{\beta} d_{\beta},\left(t_{1}^{k}+\cdots+t_{n}^{k}\right) d_{\mu}\right]=\sum_{\beta \in K_{n}, 1 \leq i \leq n} t^{\beta+k \epsilon_{i}}\left(d_{\beta}, k \epsilon_{i}\right) d_{\mu}=0 .
$$

This implies $\left(d_{\beta}, \epsilon_{i}\right)=0$ for all $i=1, \ldots, n$, i.e., $d_{\beta} \in \mathfrak{h}_{n}^{\prime}$. Thus we must have $x^{\prime} \in$ $\sum_{\beta \in K_{n}} t^{\beta} \mathfrak{h}_{n}^{\prime}$ by Claim 1. For $k<0$, we may take terms of minimal degree instead. So for any $k \in \mathbb{Z} \backslash\{0\}$, the centralizer of $\left(t_{1}^{k}+\cdots+t_{n}^{k}\right) d_{\mu}$ in $W_{\infty}$ is only

$$
\sum_{\beta \in K_{n}} \mathbb{C} t^{\beta}\left(t_{1}^{k}+\cdots+t_{n}^{k}\right) d_{\mu}+\sum_{\beta \in K_{n}} t^{\beta} \mathfrak{h}_{n}^{\prime} .
$$

Lemma 4.2. For a given $n \in \mathbb{N}$, let $\Delta$ be a 2-local derivation on $W_{\infty}$ such that $\Delta\left(d_{\mu}\right)=$ 0 . Then for any nonzero element $x=\sum_{\alpha \in S} t^{\alpha} d_{\alpha} \in W_{n}$, where $S$ is the finite subset consisting of all $\alpha \in \mathbb{Z}^{\infty}$ with $\alpha_{i}=0, i>n$ such that $d_{\alpha} \in \mathfrak{h}_{n} \backslash\{0\}$, we have

$$
\Delta(x)=\Delta\left(\sum_{\alpha \in S} t^{\alpha} d_{\alpha}\right) \in \sum_{\beta \in K_{n}} \sum_{\alpha \in S} \mathbb{C} t^{\alpha+\beta} d_{\alpha}
$$

Proof. For $x$ and $d_{\mu}$, there exists an element $a=\sum_{\beta \in \mathbb{Z}^{\infty}} t^{\beta} d_{\beta} \in W_{\infty}$, such that

$$
\Delta\left(d_{\mu}\right)=\left[a, d_{\mu}\right], \Delta(x)=\Delta\left(\sum_{\alpha \in S} t^{\alpha} d_{\alpha}\right)=\left[a, \sum_{\alpha \in S} t^{\alpha} d_{\alpha}\right]
$$

Then

$$
0=\Delta\left(d_{\mu}\right)=\left[a, d_{\mu}\right]=\left[\sum_{\beta \in \mathbb{Z}^{\infty}} t^{\beta} d_{\beta}, d_{\mu}\right]=-\sum_{\beta \in \mathbb{Z}^{\infty}} t^{\beta}\left(d_{\mu}, \beta\right) d_{\beta} .
$$

This means that $a=\sum_{\beta \in K_{n}} t^{\beta} d_{\beta}$. Thus

$$
\Delta(x)=\Delta\left(\sum_{\alpha \in S} t^{\alpha} d_{\alpha}\right)=\left[\sum_{\beta \in K_{n}} t^{\beta} d_{\beta}, \sum_{\alpha \in S} t^{\alpha} d_{\alpha}\right] \in \sum_{\beta \in K_{n}} \sum_{\alpha \in S} \mathbb{C} t^{\alpha+\beta} d_{\alpha} .
$$

Lemma 4.3. For a given $n \in \mathbb{N}$, let $\Delta$ be a 2-local derivation on $W_{\infty}$ such that

$$
\Delta\left(d_{\mu}\right)=\Delta\left(\left(t_{1}+\cdots+t_{n}\right) d_{\mu}\right)=0 .
$$

Then $\Delta\left(\left(t_{1}^{k}+\cdots+t_{n}^{k}\right) d_{\mu}\right)=0$ for all $k \in \mathbb{Z}$. 
Proof. Suppose $k \neq 0,1$. For $\left(t_{1}+\cdots+t_{n}\right) d_{\mu}$ and $\left(t_{1}^{k}+\cdots+t_{n}^{k}\right) d_{\mu}$, there exists an element $a \in W_{\infty}$ such that

$$
\begin{gathered}
0=\Delta\left(\left(t_{1}+\cdots+t_{n}\right) d_{\mu}\right)=\left[a,\left(t_{1}+\cdots+t_{n}\right) d_{\mu}\right], \\
\Delta\left(\left(t_{1}^{k}+\cdots+t_{n}^{k}\right) d_{\mu}\right)=\left[a,\left(t_{1}^{k}+\cdots+t_{n}^{k}\right) d_{\mu}\right] .
\end{gathered}
$$

Then by Lemma 4.1

$$
a=\sum_{\beta \in K_{n}} c_{\beta} t^{\beta}\left(t_{1}+\cdots+t_{n}\right) d_{\mu}+\sum_{\beta \in K_{n}, d_{\beta} \in \mathfrak{h}_{n}^{\prime}} t^{\beta} d_{\beta}, c_{\beta} \in \mathbb{C} .
$$

By Lemma 4.2 we have

$$
\Delta\left(\left(t_{1}^{k}+\cdots+t_{n}^{k}\right) d_{\mu}\right)=\left[a,\left(t_{1}^{k}+\cdots+t_{n}^{k}\right) d_{\mu}\right] \in \sum_{\beta \in K_{n}} \sum_{i=1}^{n} \mathbb{C} t^{k \epsilon_{i}+\beta} d_{\mu} .
$$

It implies that $c_{\beta}=0$, otherwise there exists a nonzero term $c_{\beta}(k-1) \mu_{1} t^{(k+1) \epsilon_{1}+\beta} d_{\mu}$ in $\Delta\left(\left(t_{1}^{k}+\cdots+t_{n}^{k}\right) d_{\mu}\right)$ not appearing in $\sum_{\beta \in K_{n}} \sum_{i=1}^{n} \mathbb{C} t^{k \epsilon_{i}+\beta} d_{\mu}$. Hence $\Delta\left(\left(t_{1}^{k}+\cdots+t_{n}^{k}\right) d_{\mu}\right)=$ 0 for all $k \in \mathbb{Z}$.

Lemma 4.4. For a given $n \in \mathbb{N}$, let $\Delta$ be a 2-local derivation on $W_{\infty}$ such that $\Delta\left(\left(t_{1}^{k}+\right.\right.$ $\left.\left.\cdots+t_{n}^{k}\right) d_{\mu}\right)=0$ for all $k \in \mathbb{Z}$. Then $\left.\Delta\right|_{W_{n}}=0$.

Proof. Take an arbitrary nonzero element $x=\sum_{\alpha \in S} t^{\alpha} d_{\alpha} \in W_{n}$, where $S$ is the finite subset consisting of all $\alpha \in \mathbb{Z}^{\infty}$ with $\alpha_{i}=0, i>n$ such that $d_{\alpha} \in \mathfrak{h}_{n} \backslash\{0\}$. Let $n_{x}$ be an index such that $\left|\alpha_{i}\right|<n_{x}$ for any $\alpha \in S$. For the fixed $k>2 n_{x}$, there is an element $a \in W_{\infty}$ such that

$$
\begin{gathered}
0=\Delta\left(\left(t_{1}^{k}+\cdots+t_{n}^{k}\right) d_{\mu}\right)=\left[a,\left(t_{1}^{k}+\cdots+t_{n}^{k}\right) d_{\mu}\right] \\
\Delta\left(\sum_{\alpha \in S} t^{\alpha} d_{\alpha}\right)=\left[a, \sum_{\alpha \in S} t^{\alpha} d_{\alpha}\right] .
\end{gathered}
$$

Then by Lemma 4.1

$$
a=\sum_{\beta \in K_{n}} c_{\beta} t^{\beta}\left(t_{1}^{k}+\cdots+t_{n}^{k}\right) d_{\mu}+\sum_{\beta \in K_{n}, d_{\beta} \in \mathfrak{h}_{n}^{\prime}} t^{\beta} d_{\beta}, c_{\beta} \in \mathbb{C} .
$$

By Lemma 4.2, we have

$$
\Delta\left(\sum_{\alpha \in S} t^{\alpha} d_{\alpha}\right)=\left[a, \sum_{\alpha \in S} t^{\alpha} d_{\alpha}\right] \in \sum_{\beta \in K_{n}} \sum_{\alpha \in S} \mathbb{C} t^{\alpha+\beta} d_{\alpha} .
$$

It implies that $c_{\beta}=0$, otherwise there exists some $t_{i}(1 \leq i \leq n)$ whose degree is more than $n_{x}$ for any term in $\Delta\left(\sum_{\alpha \in S} t^{\alpha} d_{\alpha}\right)$. So $\left.\Delta\right|_{W_{n}}=0$.

Theorem 4.5. Any 2-local derivation on $W_{\infty}$ is a derivation.

Proof. Let $\Delta$ be a 2-local derivation on $W_{\infty}$. For any $x, y \in W_{\infty}$, there exists $n \in \mathbb{N}$ such that $x, y \in W_{n}<W_{\infty}$. Take an element $a \in W_{\infty}$ such that

$$
\Delta\left(d_{\mu}\right)=\left[a, d_{\mu}\right], \Delta\left(\left(t_{1}+\cdots+t_{n}\right) d_{\mu}\right)=\left[a,\left(t_{1}+\cdots+t_{n}\right) d_{\mu}\right] .
$$

Set $\Delta_{1}=\Delta-\operatorname{ad}(a)$. Then $\Delta_{1}$ is a 2-local derivation such that

$$
\Delta_{1}\left(d_{\mu}\right)=\Delta_{1}\left(\left(t_{1}+\cdots+t_{n}\right) d_{\mu}\right)=0 .
$$

By Lemma 4.3 and Lemma 4.4, it follows that $\left.\Delta_{1}\right|_{W_{n}}=0$ and $\left.\Delta\right|_{W_{n}}=\left.\operatorname{ad}(a)\right|_{W_{n}}$. Then

$$
\begin{aligned}
& \Delta(c x+y)=\operatorname{ad}(a)(c x+y)=\operatorname{cad}(a)(x)+\operatorname{ad}(a)(y)=c \Delta(x)+\Delta(y), \forall c \in \mathbb{C}, \\
& \Delta([x, y])=\operatorname{ad}(a)([x, y])=[\operatorname{ad}(a)(x), y]+[x, \operatorname{ad}(a)(y)]=[\Delta(x), y]+[x, \Delta(y)] .
\end{aligned}
$$


Therefore $\Delta$ is a derivation.

\section{2-LOCAL DERIVATIONS ON $W_{n}^{+}, W_{n}^{++}, W_{\infty}^{+}$AND $W_{\infty}^{++}$}

For $n \in \mathbb{N}$, we have the Witt algebra $W_{n}^{+}=\operatorname{Der}\left(\mathbb{C}\left[t_{1}, t_{2}, \cdots, t_{n}\right]\right)$ which is a subalgebra of $W_{n}$. We use $\mathfrak{h}_{n}$ to denote the Cartan subalgebra of $W_{n}$ which is also a Cartan subalgebra (not unique) of $W_{n}^{+}$. We know that

$$
W_{n}^{+}=\sum_{\alpha \in \mathbb{Z}_{+}^{n}} t^{\alpha} \mathfrak{h}_{n}+\sum_{i=1}^{n} \mathbb{C} t_{i}^{-1} d_{i} .
$$

Furthermore $W_{n}^{+}$has a subalgebra

$$
W_{n}^{++}=\sum_{\alpha \in \mathbb{Z}_{+}^{n}} t^{\alpha} \mathfrak{h}_{n} .
$$

It is well-known that $W_{n}^{+}$is a simple Lie algebra, but $W_{n}^{++}$is not.

Similarly, the Witt algebra $W_{\infty}^{+}=\operatorname{Der}\left(\mathbb{C}\left[t_{1}, t_{2}, \cdots,\right]\right)$ is a subalgebra of $W_{\infty}$. We use $\mathfrak{h}_{\infty}$ to denote the Cartan subalgebra of $W_{\infty}$ which is also a Cartan subalgebra (not unique) of $W_{\infty}^{+}$. We know that

$$
W_{\infty}^{+}=\sum_{\alpha \in \mathbb{Z}_{+}^{\infty}} t^{\alpha} \mathfrak{h}_{\infty}+\sum_{i=1}^{\infty} \mathbb{C} t_{i}^{-1} d_{i} .
$$

Furthermore $W_{\infty}^{+}$has a subalgebra

$$
W_{\infty}^{++}=\sum_{\alpha \in \mathbb{Z}_{+}^{\infty}} t^{\alpha} \mathfrak{h}_{\infty} .
$$

It is well-known that $W_{\infty}^{+}$is a simple Lie algebra, but $W_{\infty}^{++}$is not.

From Proposition 4.1 and Theorem 4.3 in [7] we know that any derivation on $W_{n}^{+}$ (resp. $W_{\infty}^{+}$) is inner (resp. locally inner). Using same arguments as the proof of Proposition 3.3 in [8] we can show that any derivation on $W_{n}^{++}\left(\right.$resp. $\left.W_{\infty}^{++}\right)$is inner (resp. locally inner).

Hence, the proofs and conclusions with slight modifications in Section 3 (resp. Section 4) are applicable to $W_{n}^{+}$and $W_{n}^{++}$(resp. $W_{\infty}^{+}$and $W_{\infty}^{++}$). It is routine to verify this. We omit the details and directly state the following theorem.

Theorem 5.1. Let $n=1,2, \cdots, \infty$. Then every 2-local derivation on the Witt algebra $W_{n}^{+}$or $W_{n}^{++}$is a derivation.

Acknowledgments. This research is partially supported by NSFC (11871190) and NSERC (311907-2015). The authors are grateful to the referee for some good suggestions and for providing [3] where the results in our paper for $W_{n}$ and $W_{n}^{+}$were also obtained using different methods.

\section{REFERENCES}

[1] Sh. A. Ayupov, K. K. Kudaybergenov, I. S. Rakhimov, 2-Local derivations on finite-dimensional Lie algebras, Linear Algebra and its Applications, 474, (2015), 1-11.

[2] Sh. A. Ayupov, B. B. Yusupov, 2-Local derivations on infinite-dimensional Lie algebras, Journal of Algebra and Its Applications, doi.org/10.1142/S0219498820501005.

[3] Sh. A. Ayupov, K. K. Kudaybergenov, B B. Yusupov, 2-Local derivations on generalized Witt algebras, Linear and Multilinear Algebra, 10.1080/03081087.2019.1708846. 
[4] Y. Billig, V. Futorny, Classification of irreducible representations of Lie algebra of vector fields on a torus, J. Reine Angew. Math., 720, (2016), 199-216.

[5] Y. Billig, A. Molev, R. Zhang, Differential equations in vertex algebras and simple modules for the Lie algebra of vector fields on a torus, Adv. Math., 218, (2008), no.6, 1972-2004.

[6] E. Cartan, Les groupes de transformations continus, infinis, simples, (French) Ann. Sci. École Norm. Sup., 26, (1909), no.3, 93-161.

[7] D. Z. Djokovic, K. Zhao, Generalized Cartan type $W$ Lie algebras in characteristic zero, J. Algebra, 195, (1997), 170-210.

[8] D. Z. Djokovic, K. Zhao, Derivations, isomorphisms and second cohomology of generalized Witt algebra, Trans. Amer. Math. Soc., 350, (1998), no.2, 643-664.

[9] X. Guo, G. Liu, R. Lu, K. Zhao, Simple Witt modules that are finitely generated over the Cartan subalgebra, Moscow Math. J., 20, (2020), 43-65.

[10] J. Patera, H. Zassenhaus, The higher rank Virasoro algebras, Comm. Math. Phys., 136, (1991), $1-14$.

[11] P. Šemrl, Local automorphisms and derivations on B(H), Proc. Amer. Math. Soc., 125, (1997), $2677-2680$.

[12] B. B. Yusupov, 2-local derivations on Witt algebras, Uzbek Math. J., 125, (2018), 160-166.

School of Mathematical Sciences, Hebei Normal University, Shijiazhuang 050016, HeBei, China

E-mail address: yueqiangzhao@163.com

Mathematics Postdoctoral Research Center, Hebei Normal University, ShijiaZhuang 050016, Hebei, China

E-mail address: chenyang1729@hotmail.com

Department of Mathematics, Wilfrid laurier University, Waterloo, On, Canada N2L 3C5, and School of Mathematical Sciences, Hebei Normal University, Shijiazhuang 050016, HeBei, China

E-mail address: kzhao@wlu.ca 